J. Clin. Chem. Clin. Biochem.

Vol. 15, 1977, pp. 375-379

\title{
Wirkung von Spirolactonen auf die Ausscheidung der fraktionierten 17-Oxosteroide und von Pregnandiol im Urin
}

\author{
Von W. R. Külpmann $\left.{ }^{1}\right)$, B. Spiegelhalder $\left.{ }^{2}\right), C h$. Wilk und H. Breuer \\ Institut für Klinische Biochemie der Universität Bonn
}

(Eingegangen 10. September 1976/23. Februar 1977)

\begin{abstract}
Zusammenfassung: Es wurde die Wirkung von Spirolactonen auf die Ausscheidung der fraktionierten 17-0xosteroide (Androsteron, Ätiocholanolon, Dehydroepiandrosteron und 11-Oxoätiocholanolon) sowie von Pregnandiol im Urin unter verschiedenen Versuchsbedingungen untersucht. Die Bestimmung der 17-Oxosteroide und von Pregnandiol erfolgte nach dünnschichtchromatographischer Vorreinigung mit einer gaschromatographischen Methode. Elf männliche Versuchspersonen (Alter 21-34 Jahre) erhielten an sieben aufeinanderfolgenden Tagen jeweils $200 \mathrm{mg}$ Spironolacton (Aldactone) oral. Fünf Weibliche, nicht schwangere Versuchspersonen (Alter 20-35 Jahre) erhielten an drei aufeinanderfolgenden Tagen jeweils $600 \mathrm{mg}$ Kaliumcanrenoat (Aldactone pro injectione) i. v. Zehn Schwangere (28.-39. Schwangerschaftswoche; Alter 22-33 Jahre) erhielten ebenfalls Kaliumcanrenoat in der oben angegebenen Dosierung.
\end{abstract}

Bei den männlichen Versuchspersonen war unter Verabreichung von Spironolacton die Ausscheidung der fraktionierten 17-Oxosteroide und von Pregnandiol signifikant emiedrigt. Bei den nicht schwangeren Frauen wurde die Ausscheidung von Ätiocholanolon und Dehydroepiandrosteron, bei den Schwangeren die Ausscheidung von Pregnandiol und Dehydroepiandrosteron durch Kaliumcanrenoat vermindert. Diese Befunde legen den Schluß nahe, daß die verminderte Steroidausscheidung im Urin auf einer Hemmung steroidmetabolisierender Enzyme durch Spirolactone beruht. Als Angriffsort von Spirolactonen wird die Umwandlung von Cholesterin in Pregnenolon diskutiert.

\section{Effect of spirolactones on the urinary excretion of individual 17-oxosteroids and of pregnanediol in man}

Summary: The effect of spirolactones on the urinary excretion of individual 17-oxosteroids and of pregnanediol in man was investigated under various conditions. After purification by thin-layer chromatography, the 17-oxosteroids and pregnanediol were determined by gas chromatography. Eleven male subjects (age 21-34 years) received a daily dose of $200 \mathrm{mg}$ spironolactone (Aldactone) orally on seven consecutive days. Five female subjects (age 20-35 years) and ten pregnant women (age 20-35 years; 28 th. -39 th. week of gestation) were given a daily intravenous injection of $600 \mathrm{mg}$ potassium canrenoate (Aldactone pro injectione) on three consecutive days.

In the male subjects, the excretion of individual 17-oxosteroids and of pregnanediol was significantly reduced during administration of spironolactone. In the nonpregnant female subjects, the excretion of etiocholanolone and dehydroepiandrosterone was diminished, whereas in the pregnant women, the excretion of pregnanediol and dehydroepiandrosterone was decreased during treatment with potassium canrenoate. These results suggest that the reduced urinary excretion of steroids may be due to an inhibition of steroid metabolising enzymes by spirolactones. It seems likely that spirolactones affect the enzymatic conversion of cholesterol to pregnenolone.

\section{Einführung}

Vor mehreren Jahren wurde in unserem Laboratorium festgestellt, daß die Ausscheidung der 17-Oxosteroide und von Pregnandiol im Urin bei Schwangeren nach Verabreichung eines Aldosteronantagonisten erniedrigt ist $(1,2)$. Auch bei männlichen Versuchșpersonen wurde eine verminderte Ausscheidung der Gesamt-17-0xo: steroide unter Spirolactonen beobachtet $(3,4)$; ebenso war die Konzentration von Testosteron im Plasma von spirolactonbehandelten Männern erniedrigt (3, 5, 6, 7). Die Ursachen für die genannten Veränderungen im Urin und Plasma sind bis heute nicht geklärt. Die bisher ermittelten Daten über das Verhalten von Androstendion,

1) Jetzige Anschrift: Institut für Klinische Chemie der Medizinischen Hochschule Hannover, Karl-Wiechert-Allee 9, D-3000 Hannover.

2) Jetzige Anschrift: Deutsches Krebsforschungszentrum, Institut für Toxikologie und Chemotherapie, Im Neuenheimer Feld, D-6900 Heidelberg. 
Lutropin und Follitropin sind widersprüchlich. So wurden zum Teil keine Veränderungen $(3,5)$, zum Teil Verminderungen (7) der Androstendion-Konzentration im Blut festgestellt. Einige Autoren fanden erhöhte Lutropin-Konzentrationen im Blut (8), andere keine Veränderungen von Lutropin und Follitropin (5) und wieder andere verminderte Werte für Lutropin (4) unter Behandlung mit Spirolactonen.

Unter diesen Umständen erschien es wünschenswert, die früher festgestellte Verminderung der 17-Oxosteroidund Pregnandiolausscheidung mit einer spezifischen Methode zu überprüfen, denn es war nicht auszuschließen, daß die kolorimetrische Gruppenbestimmung der 17-Oxosteroide durch Spirolactone und ihre Metaboliten gestört wird. Darüber hinaus sollte durch die Bestimmung der fraktionierten 17-Oxosteroide versucht werden, einen Einblick in den Mechanismus der Spirolactonwirkung auf den Steroidstoffwechsel zu gewinnen. Die Ergebnisse lassen vermuten, daß Spirolactone vornehmlich die Biogenese von Steroidhormonen in der Nebenniere und im Hoden hemmen.

\section{Methoden}

\section{Versuchspersonen}

Die männlichen Versuchspersonen waren zwischen 21 und 34 Jahre alt. Auf Grund ihrer Anamnese und klinisch-chemischer Untersuchungen (Aspartat-Aminotransferase, Alanin-Aminotransferase, Cholinesterase, alkalische Phosphatase, LactatDehydrogenase, Bilirubin im Serum, Gesamteiweiß, Cholesterin) konnten die Probanden als gesund gelten. Sie erhielten täglich $200 \mathrm{mg}$ Spironolacton (Aldactone) oral an sieben aufeinanderfolgenden Tagen. Am Tage vor der Behandlung sowie am 7. Tag der Behandlung wurden die $24 \mathrm{~h}$-Urine gesammelt.

Die nicht schwangeren weiblichen Versuchspersonen waren zwischen 20 und 35 Jahre alt. Sie konnten auf Grund der oben angegebenen Kriterien als gesund gelten und nahmen keine oralen Contraceptiva ein. Die etwa gleich alten Schwangeren befanden sich in der 28.-39. Schwangerschaftswoche und zeigten keine Funktionsstörungen der Leber und des Endokriniums ${ }^{3}$ ); alle Frauen erhielten täglich $600 \mathrm{mg}$ Kaliumcanrenoat (Aldactone pro injectione) i. v. an drei aufeinanderfolgenden Tagen. Am Tage vor der Behandlung sowie am 3. Tage der Behandlung wurden die 24-h-Urine gesammelt. Die Urine wurden mit Wasser auf $1200 \mathrm{ml}$ aufgefüllt und bis zur Aufarbeitung ohne Zusatz bei $-10^{\circ} \mathrm{C}$ aufbewahrt. Während des Untersuchungszeitraums nahmen die Probanden keinen Alkohol zu sich; auf die Einhaltung einer besonderen Diät wurde verzichtet.

Bestimmung der fraktionierten 17-Oxosteroide und von Pregnandiol

Die Steroidausscheidung im Urin wurde mit Hilfe einer spezifischen gaschromatographischen Methode bestimmt; Einzelheiten sind an anderer Stelle ausführlich beschrieben worden (9). Von jedem 24h-Urin wurden zwei Proben getrennt aufge-

3) Wegen der 1970/71 $(1,2)$ bei Schwangeren erstmals beobachteten Verminderung der Pregnandiolausscheidung unter Spirolactonen wurde, um eine Gefährdung des Fetus zu vermeiden, auf aliquote Teile der Urinproben der damaligen Versuchsreihe zurückgegriffen. Das Untersuchungsmaterial war in der Zwischenzeit tiefgefroren aufbewahrt worden. Die Analysen wurden 1973 durchgeführt. arbeitet und der gaschromatographischen Bestimmung an zwei verschiedenen Säulen unterworfen. Die eine Säule war mit 3\% OV-225 und 3\% OV-210 (auf Chromosorb WHP, gemischt im Verhältnis 1,8:1,0) (vgl. (9)), die andere mit 3\% PPE-21 (auf Chro mosorb WHP) gefüllt. Die beiden Säulenfüllungen mit unterschiedilicher Polarität wurden im Hinblick auf mögliche Störungen durch Metaboliten von Spirolactonen verwendet. Die gaschromatographischen Bestimmungen erfolgten mit einem Microtek MT 220 (Techmation, Düsseldorf) und einem Aerograph 1800 (Varian, Darmstadt). Die Rechenoperationen wurden mit einem Tischrechner (Olivetti „Programma“, Olivetti, Frankfurt/Main), die statistischen Berechnungen mit Hilfe des „Statistics Calculator" (Hewlett-Packard, Loveland, Co., U.S.A.) durchgefürt.

\section{Ergebnisse}

\section{Männliche Versuchspersonen}

Bei allen untersuchten männlichen Normalpersonen war die Ausscheidung der einzelnen 17-0xosteroide und von Pregnandiol unter Behandlung mit Spironolacton (Aldactone) signifikant vermindert (Tab. 1-3). Die durchschnittliche Abnahme betrug für Androsteron $15 \%$, für Ätiocholanolon $23 \%$, für Dehydroepiandro-

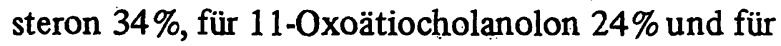
Pregnandiol 24\%. Die Prüfung auf Signifikanz wurde mit Hilfe des einseitigen t-Tests für gepaarte Daten durchgeführt (Tab. 4).

\section{Nicht schwangere Frauen}

Bei den nicht schwangeren Frauen konnte nach Behandlung mit einem Spirolacton (Kaliumcanrenoat) lediglich für die Urinausscheidung von Dehydroepiandrosteron und Ätiocholanolon eine Abnahme festgestellt werden. Sie betrug im Mittel 25\% für Dehydroepiandrosteron $(0,1>\ddot{P}>0,05 ; \mathrm{N}=5)$ und $56 \%$ für

Tab. 1. Ausscheidung von Androsteron (A) und Ätiocholanolon (Ä) im Urin in $\mu \mathrm{mol} / 24 \mathrm{~h}$ bei elf männlichen Versuchspersonen vor (I) und nach (II) siebentägiger Behandlung mit Spironolacton $(200 \mathrm{mg} / \mathrm{d})$. Die Werte sind Mittelwerte aus jeweils zwei Proben (entsprechend acht gaschromatographischen Einzelbestimmungen). Die Änderungen der Ausscheidung $(\Delta \%)$ sind in Prozent angegeben.

\begin{tabular}{|c|c|c|c|c|c|c|}
\hline \multirow[b]{2}{*}{$\begin{array}{l}\text { Vers. } \\
\text { Pers. }\end{array}$} & \multicolumn{2}{|c|}{$\begin{array}{l}\text { Ausscheidung von } \\
\text { Androsteron } \\
\text { ( } \mu \text { mol/24 h) }\end{array}$} & \multicolumn{2}{|c|}{$\begin{array}{l}\text { Ätiocholanolon } \\
(\mu \mathrm{mol} / 24 \mathrm{~h})\end{array}$} & \multicolumn{2}{|c|}{$\begin{array}{l}\text { Änderung der } \\
\text { Ausscheidung } \\
\text { A } \ddot{A}\end{array}$} \\
\hline & I & II & I & II & $\Delta \%$ & $\Delta \%$ \\
\hline 1 & 11,6 & 10,2 & 6,4 & 4,1 & -12 & -36 \\
\hline 2 & 6,5 & 5,2 & 3,9 & 3,0 & -20 & -23 \\
\hline 3 & 5,1 & 4,4 & 11,6 & 7,9 & -14 & -32 \\
\hline 4 & 6,7 & 5,6 & 6,1 & 5,8 & -16 & -5 \\
\hline 5 & 8,2 & 7,6 & 2,2 & 1,9 & -7 & -14 \\
\hline 6 & 8,4 & 7,9 & 4,1 & 3,6 & -6 & -12 \\
\hline 7 & 9,4 & 8,2 & 4,3 & 3,9 & -13 & $\begin{array}{r}12 \\
-9\end{array}$ \\
\hline .8 & 12,9 & 10,1 & 6,0 & 5,3 & -22 & -12 \\
\hline 9 & 9,3 & 6,6 & 6,9 & 3,0 & -29 & -57 \\
\hline 10 & 4,9 & 4,4 & 8,8 & 6,1 & -10 & -31 \\
\hline 11 & 10,1 & 8,9 & 6,5 & 5,9 & -12 & -19 \\
\hline
\end{tabular}


Ätiocholanolon $(0,0125>P>0,01 ; N=5)$. Hingegen zeigte die Ausscheidung von Androsteron und von Pregnandiol keine statistisch signifikanten Veränderungen.

\section{Schwangere Frauen}

Die Ausscheidung von Pregnandiol im Urin schwangerer Frauen wurde durch Spirolacton (Kaliumcanrenoat) deutlich vermindert (Tab. 5). Die Abnahme betrug im Mittel 52\%. Ähnliches gilt für die Ausscheidung von Dehydroepiandrosteron; hier betrug die mittlere Hemmung $10 \%$. Die Signifikanzprüfung ergab folgende Werte: $\mathrm{P}<0,0005$ für Pregnandiol $(\mathrm{N}=10)$ und $0,1>P>0,05$ für Dehydroepiandrosteron $(N=4)$.

Tab. 2. Ausscheidung von Dehydroepiandrosteron (DHEA) in $\mu \mathrm{mol} / 24 \mathrm{~h}$ und Pregnandiol $(P)$ in nmol/24 h im Urin bei elf männlichen Versuchspersonen vor (I) und nach (II) siebentägiger Behandlung mit Spironolacton (200 $\mathrm{mg} / \mathrm{d})$. Die Werte sind Mittelwerte aus jeweils zwei Proben (entsprechend acht gaschromatographischen Einzelbestimmungen). Die Ânderungen der Ausscheidung $(\Delta \%)$ sind in Prozent angegeben.

\begin{tabular}{|c|c|c|c|c|c|c|}
\hline \multirow[b]{2}{*}{$\begin{array}{l}\text { Vers. } \\
\text { Pers. }\end{array}$} & \multicolumn{2}{|c|}{$\begin{array}{l}\text { Ausscheidung von } \\
\text { Dehydroepiandro- } \\
\text { steron } \\
\mu \mathrm{mol} / 24 \mathrm{~h}\end{array}$} & \multicolumn{2}{|c|}{$\begin{array}{l}\text { Ausscheidung von } \\
\text { Pregnandiol } \\
\mathrm{nmol} / 24 \mathrm{~h}\end{array}$} & \multicolumn{2}{|c|}{$\begin{array}{l}\text { Änderung der } \\
\text { Ausscheidung }\end{array}$} \\
\hline & I & II & I & II & $\Delta \%$ & $\Delta \%$ \\
\hline $\begin{array}{r}1 \\
2 \\
3 \\
4 \\
5 \\
6 \\
7 \\
8 \\
9 \\
10 \\
11\end{array}$ & $\begin{array}{r}0,8 \\
0,6 \\
1,3 \\
2,9 \\
12,4 \\
4,3 \\
1,5 \\
0,8 \\
7,1 \\
1,4 \\
19,3\end{array}$ & $\begin{array}{r}0,5 \\
0,5 \\
0,9 \\
1,7 \\
6,0 \\
4,1 \\
1,0 \\
0,7 \\
2,2 \\
0,7 \\
14,5\end{array}$ & $\begin{array}{r}660 \\
590 \\
690 \\
720 \\
630 \\
1720 \\
750 \\
1720 \\
2060 \\
840 \\
1280\end{array}$ & $\begin{array}{r}470 \\
470 \\
530 \\
630 \\
590 \\
1000 \\
660 \\
1340 \\
880 \\
590 \\
1060\end{array}$ & $\begin{array}{l}-38 \\
-17 \\
-31 \\
-41 \\
-52 \\
-5 \\
-33 \\
-13 \\
-69 \\
-50 \\
-25\end{array}$ & $\begin{array}{l}-29 \\
-20 \\
-23 \\
-13 \\
-6 \\
-42 \\
-12 \\
-22 \\
-52 \\
-30 \\
-17\end{array}$ \\
\hline
\end{tabular}

Tab. 3. Ausscheheidung von 11-Oxoätiocholanolon im Urin in $\mu \mathrm{mol} / 24 \mathrm{~h}$ bei sechs männlichen Versuchspersonen vor und nach siebentägiger Behandlung mit Spironolacton $(200 \mathrm{mg} / \mathrm{d})$. Die Werte sind Mittelwerte aus jeweils zwei Proben (entsprechend acht gaschromatographischen Einzelbestimmungen).

\begin{tabular}{|c|c|c|c|}
\hline \multicolumn{3}{|c|}{$\begin{array}{l}\text { Ausscheidung von } \\
11-\text { Oxoätiocholanolon } \\
\mu \mathrm{mol} / 24 \mathrm{~h}\end{array}$} & \multirow{2}{*}{$\begin{array}{l}\text { Ânderung der } \\
\text { Ausscheidung } \\
\Delta \%\end{array}$} \\
\hline $\begin{array}{l}\text { Vers, } \\
\text { Pers. }\end{array}$ & $\begin{array}{l}\text { vơt Ver- } \\
\text { abreichung } \\
\text { von Spirono- } \\
\text { lactonn }\end{array}$ & $\begin{array}{l}\text { nach Ver- } \\
\text { abreieichung } \\
\text { von Spirono- } \\
\text { lacton }\end{array}$ & \\
\hline $\begin{array}{l}1 \\
2 \\
3 \\
4 \\
5 \\
6\end{array}$ & $\begin{array}{l}1,02 \\
3,20 \\
0,69 \\
1,35 \\
1,48 \\
3,16\end{array}$ & $\begin{array}{l}0,82 \\
2,71 \\
0,63 \\
0,99 \\
0,63 \\
2,73\end{array}$ & $\begin{array}{l}-20 \\
-15 \\
-\quad 9 \\
-27 \\
-57 \\
-14\end{array}$ \\
\hline
\end{tabular}

Tab. 4. Signifikanzprüfung der Wirkung von Spironolacton (Aldactone) auf die Ausscheidung verschiedener 17Oxosteroide und Pregnandiol im Urin männlicher Versuchspersonen mit Hilfe des einseitigen $t$-Tests für gepaarte Daten.

\begin{tabular}{llr}
\hline Steroid & $\begin{array}{l}\text { Ergebnis der Signifikanz- } \\
\text { prüfung }\end{array}$ & $\mathrm{N}$ \\
\hline Androsteron & \multicolumn{1}{c}{$\mathrm{P}<0,0005$} & 11 \\
Ätiocholanolon & $0,0025>\mathrm{P}>0,0005$ & 11 \\
Dehydroepiandrosteron & $0,025>\mathrm{P}>0,0125$ & 11 \\
11-Oxoätiocholanolon & $0,0125>\mathrm{P}>0,01$ & 6 \\
Pregnandiol & $0,01 \quad>\mathrm{P}>0,005$ & 11 \\
\hline
\end{tabular}

Tab. 5. Ausscheidung von Pregnandiol im Urin in $\mu \mathrm{mol} / 24 \mathrm{~h}$ bei Schwangeren (28.-39. Schwangerschaftswoche) vor und nach dreitägiger Behandlung mit Kaliumcanrenoat $(600 \mathrm{mg} / \mathrm{d})$. Die Werte sind Mittelwerte aus jeweils zwei Proben (entsprechend acht gaschromatographischen Einzelbestimmungen).

\begin{tabular}{|c|c|c|c|}
\hline \multicolumn{3}{|c|}{$\begin{array}{l}\text { Ausscheidung von Pregnandiol } \\
(\mu \mathrm{mol} / 24 \mathrm{~h})\end{array}$} & \multirow{2}{*}{$\begin{array}{l}\text { Änderung der } \\
\text { Ausscheidung } \\
\Delta \%\end{array}$} \\
\hline $\begin{array}{l}\text { Vers. } \\
\text { Pers. }\end{array}$ & $\begin{array}{l}\text { vor Ver- } \\
\text { abreichung } \\
\text { von K-Canrenoat }\end{array}$ & $\begin{array}{l}\text { nach Ver- } \\
\text { abreichung } \\
\text { von K-Canrenoat }\end{array}$ & \\
\hline $\begin{array}{r}1 \\
2 \\
3 \\
4 \\
5 \\
6 \\
7 \\
8 \\
9 \\
10\end{array}$ & $\begin{array}{l}31,3 \\
62,5 \\
53,1 \\
46,9 \\
25,0 \\
62,5 \\
40,0 \\
53,1 \\
71,9 \\
25,0\end{array}$ & $\begin{array}{l}12,5 \\
31,3 \\
34,4 \\
10,9 \\
12,5 \\
33,4 \\
23,8 \\
25,0 \\
31,3 \\
12,5\end{array}$ & $\begin{array}{l}-60 \\
-50 \\
-35 \\
-77 \\
-50 \\
-47 \\
-41 \\
-53 \\
-56 \\
-50\end{array}$ \\
\hline
\end{tabular}

\section{Diskussion}

Die vorliegenden Ergebnisse, die mit einem spezifischen gaschromatographischen Verfahren gewonnen wurden, bestätigen die bereits früher mit kolorimetrischen Methoden erhaltenen Befunde, wonach die Ausscheidung der Gesamt-17-Oxosteroide im Urin unter Verabreichung von Spirolactonen vermindert ist (1-4). Darüber hinaus konnte gezeigt werden, daß diese Hemmung bei männlichen Versuchspersonen alle hier untersuchten fraktionierten 17-Oxosteroide betrifft. Bei den nicht schwangeren Frauen waren die Ergebnisse etwas uneinheitlicher; möglicherweise ist dies auf cyclusbedingte Schwankungen zurückzuführen. Versuche, in denen für die Dauer eines Cyclus entsprechende Dosen eines Spirolactons verabreicht werden, könnten ein eindeutigeres Bild vermitteln. Bei den Schwangeren war eine Hemmung nur für die Ausscheidung von Pregnandiol und Dehydroepiandrosteron nachweisbar. Der hier geführte spezifische Nachweis einer verminderten Ausscheidung fraktionierter 
17-Oxosteroide nach Spirolacton-Verabreichung schließt die Möglichkeit, daß die Hemmung methodisch vorgetäuscht ist, weitgehend aus.

Die Tatsache, daß die Ausscheidung der einzelnen 17Oxosteroide in unterschiedlichem und wechselndem Ausmaß durch Spirolactone beeinflußt wird, spricht gegen eine grundsätzliche Änderung des Steroidstoffwechsels. Die Verminderung der Ausscheidung von Androsteron und Ätiocholanolon - bei gleichzeitig verminderter Ausscheidung von Dehydroepiandrosteron ist sowoil mit einer verminderten Produktion von Testosteron in den Testes als auch mit einer erhöhten metabolischen Clearancerate für Testosteron vereinbar. Für eine Einschränkung der Testosteronproduktion sprechen die Ergebnisse von Stripp et al. (10), die bei versciliedenen Tieren (Hund, Ratte, Maus, Meerschweinchen) eine verminderte Konzentration von Testosteron in der vena testicularis unter Dauertropfinfusion von Spirolactonen gemessen haben. Für eine erhöhte metabolische Clearancerate könnte die Beobachtung von Dymling \& Hökfelt (5) sprechen, die bei zwei Patienten nach i. m. Verabreichung von Testosteronpropionat eine beschleunigte Abnahme der Testosteronkonzentration im Plasma feststellten, wenn gleichzeitig Spirolactone verabreicht wurden.

Auch die verminderte Ausscheidung von Dehydroepiandrosteron ließe sich durch eine eingeschränkte Produktion des Steroids in der Nebennierenrinde erklären. In der Tat fanden Erbler (11) und Menard et al. (12) eine Hemmung adrenaler Hydroxylasen durch Spirolactone.

Die hier erhobenen Befunde sprechen dafür, daß die Bildung von Progesteron, dessen Hauptmetabolit Pregnandiol ist, sowie die von Testosteron und Dehydroepiandrosteron durch Spirolactone vermindert wird. Die Hemmung der Steroidbiosynthese könnte auf der Stufe der Umwandlung von Cholesterin in Pregnenolon erfolgen. An dieser Stelle greifen auch die meisten anderen Steuerungsprinzipien der Steroidbiosynthese ein. Mit großer Wahrscheinlichkeit wird die Steroidproduktion sowohl in der Nebennierenrinde als auch in den Testes beeinträchtigt. Für diese Annahme spricht die Beobachtung, daß das im Urin ausgeschiedene Dehydroepiandrosteron hauptsächlich adrenalen Ursprungs ist, im vorliegenden Falle also die Hemmung in der Nebennierenrinde zu suchen ist. Andererseits ist die Abnahme der Ausscheidung von Androsteron und Ätiocholanolon unter Spirolactonen so groß, daß sie nicht allein durch eine verminderte periphere Umwandlung von Androstendion zu Testosteron erklärt werden kann. Diese Vermutung wird durch die Befunde anderer Autoren $(3,5,6,7)$ unterstützt, die eine deutliche Abnahme der Testosteronkonzentration im Plasma nach Verabreichung von Spirolactonen feststellten. Die von Menard et al. (13) in vitro beobachtete Hemmung der Umwandlung von Progesteron in Testosteron könnte zusätzlich zu einer Produktionsverminderung dieses Androgens führen. Unabhängig von der Verminderung der Testosteronproduktion beruht ein Teil der anti-androgenen Wirkung der Spirolactone auf einer direkten kompetitiven Hemmung von $5 \alpha$-Dihydrotestosteron am Rezeptor (14). Eine Erhöhung der metabolischen Clearancerate, wie sie für Barbiturate als Folge der Induktion mikrosomaler Leberenzyme durch Spirolactone gut belegt ist $(15,16,17)$, konnte für Steroidhormone bisher nicht schlüssig bewiesen werden. Nach unseren Ergebnissen müßten in diesem Fall besondere Stoffwechselwege beschritten werden.

Faßt man die bisherigen Befunde zusammen, so kann eine direkte Wirkung von Spirolactonen auf periphere steroidbildende Organè als weitgehend gesichert gelten. Spirolactone sind nicht ausschließlich Aldosteronantagonisten; die Frage, ob sie auch über eine Beeinflussung hy pothalamischer/hypophysärer Zentren wirksam werden können, bleibt zunächst offen. Einige widersprüchliche Ergebnisse dürften dadurch zu erklären sein, daß bestimmte Effekte der Spirolactone offenbar von der Dosierung sowie von anderen Versuchsbedingungen (Spezies, Geschlecht) abhängig sind.

\section{Danksagung}

Wir danken der Deutschen Forschungsgemeinschaft für eine Sachbeihilfe.

\section{Literatur}

1. Nocke, L., Breuer, H. \& Lichton, J. I. (1970), Excerptä Med. International Congress Series No. 210, 383.

2. Nocke, L., Breuer, H., Klink, R., Lichton, I. R. \& Nocke, W. (1971), Acta Endocrinol (Kbh.) Suppl. 152, 41.

3. Dymling, J. F., Nilsson, K. O. \& Hökfelt, B. (1972), Acta Endocrinol. (Kbh.) 70, 104-112.

4. Bridgman, J. F. \& Buckler, J. M. H. (1974), Br. Med. J. 3, $520-521$.

5. Dymling, J. F. \& Hökfelt, B. (1973), Acta Endọcrinol. (Kbh.) Suppl. 177, 53.
6. Erbler, H. C. (1974), Naunyn Schmiedeberg's Arch. Pharmacol. 285, 403-406.

7. Walsh, P. C. \& Siiteri, P. K. (1975), in Normal and abnormal growth of the prostate (Axelrod, L. R., ed.), Verlag C. C. Thomas, Springfield, Ill., USA.

'8. Peintikainen, P. J., Peintikainen, L. A., Huffman, D. H. \& Aravnoff, D. L. (1973), Clin. Res. 21, 472.

9. Külpmann, W. R. \& Breuer, H. (1977), dieses J. 15, $225-231$. 
10. Stripp, B., Menard, R. H., Loriaux, L., Taylor, A., Gillette, J. 14. Corvol, P., Michaud, A., Menard, J., Freifeld, M. \& Mahou\& Bartter, F. (1974), J. Clin. Invest. 53, 79 a.

deau, J. (1975), Endocrinology 97, 52-58.

11. Erbler, H. C. (1973), Naunyn Schmiedeberg's Arch. Pharmacol. 15. Soly moss, B., Classen, H. G. \& Varga, S. (1969), Proc. Soc. 277, Suppl. R 15.

12. Menard, R. H., Bartter, F. C. \& Gillette, J. R. (1976), Arch. Biochem. Biophys. 173, 395-402.

13. Menard, R. H., Stripp, B. \& Gillette, J. R. (1974), Endocrinology $94,1628-1636$.

Exp. Biol. Med. 132, 940-942.

16. Gerald, M. C. \& Feller, D. R. (1970), Biochem. Pharmacol. $19,2529-2532$.

17. Feller, D. R. \& Gerald, M. C. (1971), Biochem. Pharmacol. $20,1991-2000$.
Dr. W. R. Külpmann Institut für Klinische Chemie

der Medizinischen Hochschule Hannover

Karl-Wiechert-Allee 9

D-3000 Hannover 
